\title{
Ossifying Fibroma of the Ethmoid Sinuses: Rare Scenarios
}

\author{
${ }^{1}$ Amit Keshri, ${ }^{2}$ Kranti Bhavana, ${ }^{1}$ Sushil Kumar Aggarwal, ${ }^{3}$ Raj Kumar, ${ }^{4}$ Deepika Upadhyay, ${ }^{5}$ Rakesh Pandey \\ ${ }^{1}$ Senior Resident, Department of Neurosurgery, Sanjay Gandhi Postgraduate Institute of Medical Sciences, Lucknow, Uttar Pradesh, India \\ ${ }^{2}$ Assistant Professor, Department of Neurosurgery, Sanjay Gandhi Postgraduate Institute of Medical Sciences, Lucknow, Uttar Pradesh, India \\ ${ }^{3}$ Professor and Head, Department of Neurosurgery, Sanjay Gandhi Postgraduate Institute of Medical Sciences, Lucknow, Uttar Pradesh, India \\ ${ }^{4}$ Senior Resident, Department of Pathology, Sanjay Gandhi Postgraduate Institute of Medical Sciences, Lucknow, Uttar Pradesh, India \\ ${ }^{5}$ Professor, Department of Pathology, Sanjay Gandhi Postgraduate Institute of Medical Sciences, Lucknow, Uttar Pradesh, India
}

Correspondence: Sushil Kumar Aggarwal, Senior Resident, Department of Neurosurgery, Sanjay Gandhi Postgraduate Institute of Medical Sciences, Lucknow-226014, Uttar Pradesh, India, Phone: +91-8004904460(M) +91-7275721672(M) +91-522-2494526(O), Fax: +91-522-668973, e-mail: doc.sushil.pgi@gmail.com

\section{ABSTRACT}

Ossifying fibroma (OF) is a rare benign tumor, which is a distinct entity, despite being closely related to fibrous dysplasia, according to WHO classification of $2005 .{ }^{1}$ It is commonly found in mandible and maxilla but rarely found in ethmoid sinuses, orbit and skull base.$^{2-4}$ It commonly occurs in age group of 20 to 40 years, ${ }^{5,6}$ although rarely, it can present in all age groups. OF is more common in females than males. ${ }^{4,7}$ We describe here two cases of OF involving the ethmoid sinuses in young male patients which presented diagnostic dilemmas for us. We report these cases as the site of involvement, age group and sex of patients and diagnostic and management considerations imparted uniqueness to these cases.

Keywords: Ossifying fibroma, Ethmoid, Juvenile age group.

\section{INTRODUCTION}

Ossifying fibroma (OF) is defined as a circumscribed seldom encapsulated benign neoplasm that consists of connective tissue containing varying amount of mineralized substance. Clinically, they are slow growing tumors and painless until they enlarge enough to cause pressure symptoms or swelling or deformity in the area of occurrence. Radiologically, they are translucent opacities which expand slowly and calcification starts in center as the lesion grows. The diagnosis requires a variety of correlation of clinical, radiological and pathological features. Recurrence rate is high especially in paranasal sinuses. Wide local excision of lesion is the treatment of choice. Exact etiology of lesion is not known, but trauma is considered to be a predisposing factor for its occurrence. The involvement of skull base, ethmoid and orbit makes complete surgical excision difficult and propensity of recurrence in cases of incomplete removal further add up to the dilemma regarding the management of such cases. We have tried to address these problems in the cases reported which may also provide an insight toward the management of such cases.

\section{CASE REPORTS}

\section{Case 1}

A 14-year-old boy was referred to us with chief complaints of protrusion of right eye for 6 months. There was no history of trauma, nasal obstruction, nasal discharge or bleeding from the nose. Physical examination showed inferolateral nonaxial proptosis of right eye. Vision was normal. Nasal endoscopy revealed a globular mass in right ethmoid sinus area which was covered by mucosa and was bony hard on touch. Middle turbinate was obscured by the swelling on the right side. The nasal septum on the left side was deviated considerably.

CT scan revealed a large calcified mass in left ethmoid sinus obliterating the middle turbinate and the mass was eroding the medial wall of orbit. Superiorly, it was attached to the skull base around the cribriform plate region. Septum was pushed toward the left side (Fig. 1).

Endoscopic excision of mass was done under general anesthesia and mass was removed in piecemeal till the skull base was reached. En bloc resection was difficult due to its attachment to vital structures in the orbit and skull base. Tumor tissue attached to medial orbital wall and the skull base was left behind. Maximal removal of tumor tissue was ensured.

Histopathology showed a well-circumscribed fibroosseous lesion consisting of many acellular, basophilic spherical ossicles with presence of some focal areas of ossicles resembling psammoma bodies. The intervening stroma was moderately cellular and showed compact fibrous tissues with bland nuclear character (Fig. 2). 


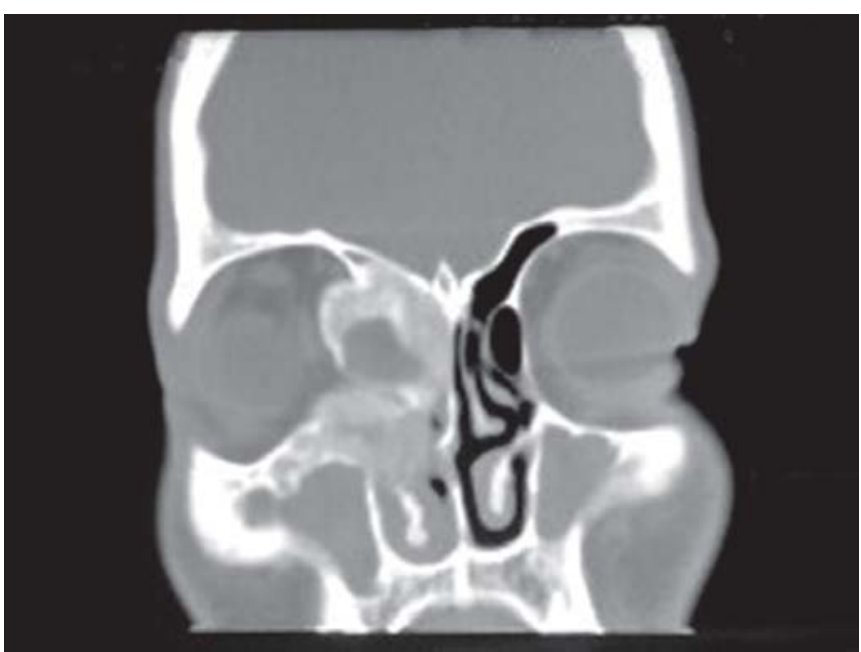

Fig. 1: CECT scan of patient showing heterogenously enhancing mixed density pattern of ossifying fibroma

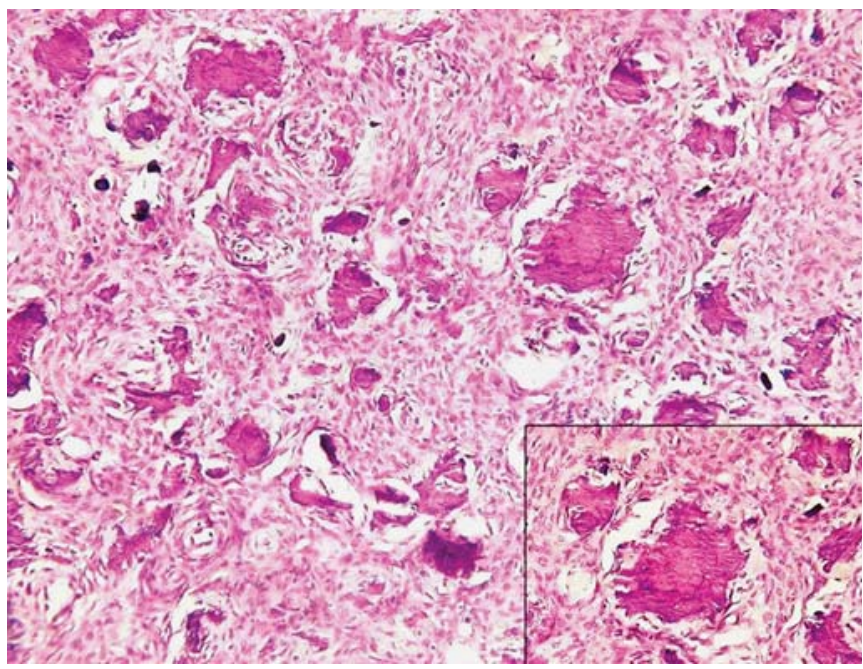

Fig. 2: Acellular, basophilic spherical to irregular ossicles with intervening stroma showing moderately cellular dense fibrous tissue

Patient was asymptomatic for 6 months but had recurrence of similar symptoms after 6 months. CT scan revealed recurrence of swelling and reexcision of mass was done again by external approach through Lynch-Howarth incision ensuring near complete removal, especially from the medial orbital wall area. Though the external ethmoidectomy approach is a traditional approach but, in this particular case, Lynch-Howarth incision gave us an excellent dissection plane between the tumor tissue and the orbital periosteum and hence reduced the chances of recurrence.

\section{Case 2}

An 18-year-old male presented to our OPD with complaints of left-sided progressive nasal obstruction for 1 year. There was no history of trauma to nose, nasal discharge or epistaxis. Physical examination of face was unremarkable and there was no proptosis. Nasal endoscopy revealed a lobular mass in the region of left ethmoid pushing the middle turbinate laterally and septum was pushed to right. CT scan revealed a large calcified mass arising from left ethmoidal roof and pushing middle turbinate medially and coming out through the middle meatus (Fig. 3). Orbital wall was normal.

Endoscopic excision of mass was done under general anesthesia. The mass was fibro-osseous in nature and could be completely scooped out from the nose till the skull base was reached. En bloc resection was not possible, hence, piecemeal removal was done endoscopically. Keeping in view the attachment of tumor mass to skull base and lateral wall of left sphenoid sinus, we were very cautious during the surgery although, we ensured complete tumor removal and normal airway patency on the involved side was achieved.

Histopathology showed a well-circumscribed and partly encapsulated fibro-osseous lesion consisting of an abundance of cellular fibrous tissue with many dispersed spherical to irregularly shaped and fused ossicles resembling psammoma bodies in many areas, along with scattered trabeculae of lamellar and woven bone. The ossicles were acellular, basophilic and showed an osteoid rim of varying width. The stromal cells were spindle shaped and contained bland looking nuclei (Fig. 4). CT scan at 6-month follow-up showed no recurrence of tumor mass, and the patient is completely asymptomatic till date (Fig. 5).

\section{DISCUSSION}

The term 'ossifying fibroma' has been used since 1927, and since 1968 cementum-containing tumors have been grouped together with this entity. ${ }^{8}$ In 1971, the World Health Organization (WHO) classified four types of such lesions:

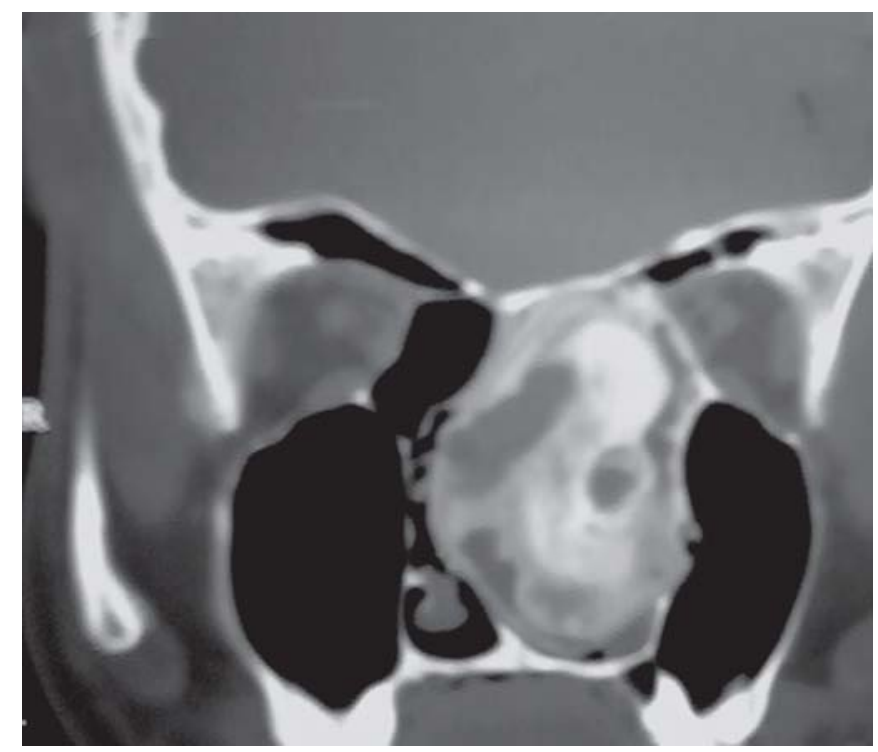

Fig. 3: Coronal cut of CECT PNS, nose and orbit showing distinct concentric lesion attached to ethmoidal roof 


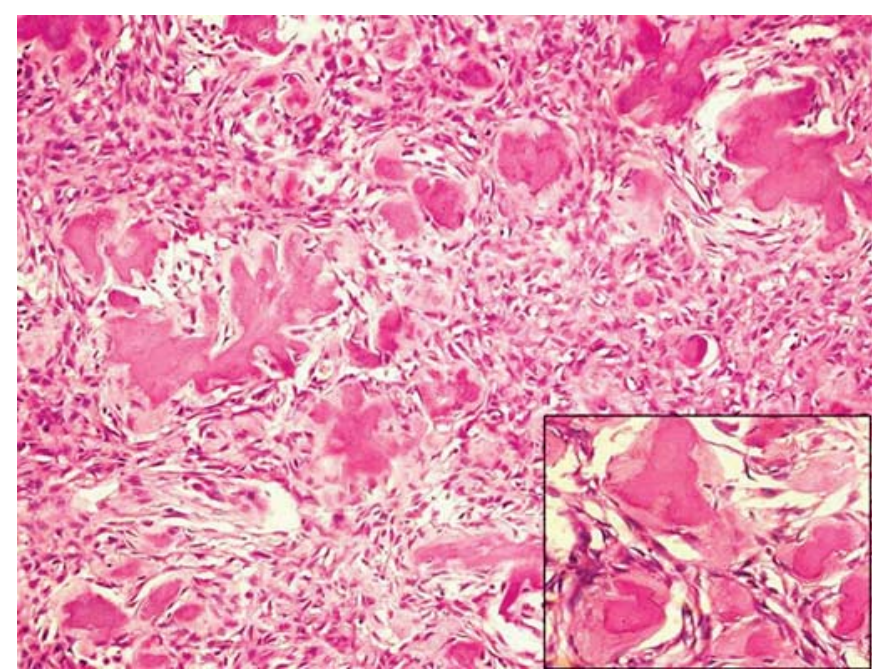

Fig. 4: Spherical to curved and fused ossicles with irregular rim of osteoid. The intervening stroma is moderately cellular and consists of densely packed fibroblasts

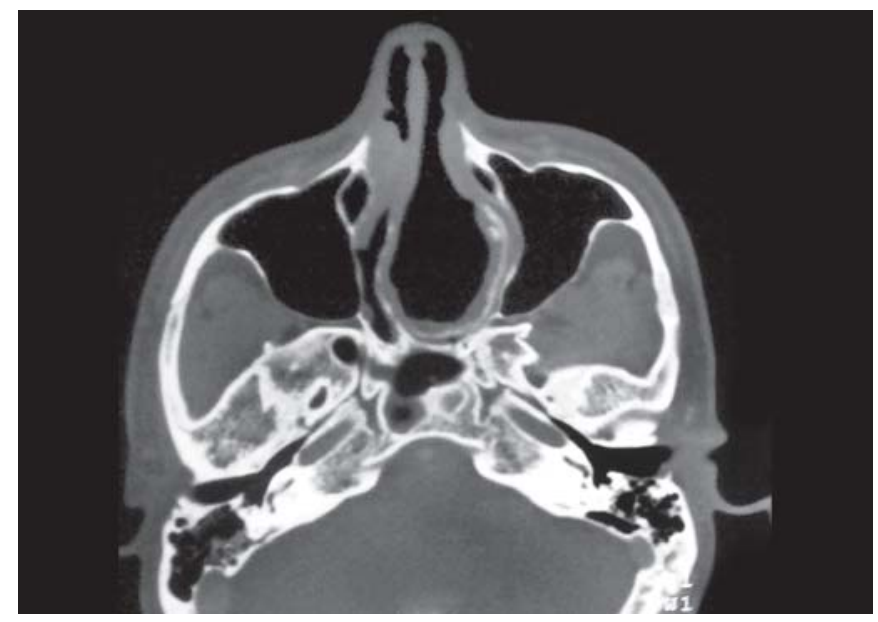

Fig. 5: Axial picture of CECT PNS showing near complete removal of left ossifying fibroma

Fibrous dysplasia, ossifying fibroma, cementifying fibroma and cemento-ossifying fibroma. ${ }^{9}$ Benign fibro-osseous lesions in the oral and maxillofacial regions were divided into osteogenic and nonneoplastic bone lesions; cementifying ossifying fibroma belonged to the former category. ${ }^{10}$ However, the term 'cementifying ossifying fibroma' was included as a synonym of ossifying fibroma in the new WHO classification in 2005. ${ }^{1}$ Cemento-ossifying fibroma consists both of cementum, like calcification and bony material. Although exact etiology of the tumor is not known, it is supposed to be of primitive mesenchymal origin. During the embryonic stage, the medial portion of nasal drift passes through the ethmoidal roof during normal transposition and a minor part of periodontal membrane is mesenchymal in origin. ${ }^{11}$ The primitive mesenchymal theory supports the occurrence of OF commonly in region of mandible followed by maxilla and ethmoid sinus and skull base.
OF as reported in literature is more commonly found in females (male:female ratio 1:2) ${ }^{4,7}$ but, on the contrary, both of our patients were male. According to the literature, OFs are more common in 3rd and 4th decades of life, ${ }^{5,6}$ but both of our patients were adolescent males presenting to us in their second decade of life. However, both the tumors had areas resembling juvenile psammomatous ossifying fibroma in variable amounts which are known to affect a younger age group. Thus, our cases signify that $\mathrm{OF}$ is not uncommon in males, and it can present in all age groups and tend to have psammomatous areas in younger patients.

Trauma has been seen as an inciting factor of occurrence, ${ }^{3,7,12}$ but both of our patients had no history of trauma to nose or paranasal sinuses. Clinically, OFs are very slow-growing tumors and do not produce any symptoms until they become large enough to compress surrounding structures or present as external swelling. In our patients, one had proptosis due to displacement of globe and another presented as nasal obstruction due to mass effect in nasal cavity. OF has been found as an incidental finding when CT or MRI is ordered for evaluation of some other disease or symptom. Meningitis and pneumocephalus have been reported as complications of OFs.

Radiologically, the initial lesions may show unilocular radiolucencies and somewhat sclerotic borders with gradual transformation to radiopacity. CT scan clarifies wellcircumscribed lesions as seen in our cases. This characteristically distinguishes FO from fibrous dysplasia. ${ }^{3}$ The central area consists of nonhomogenous matrix with 'ground-glass' opacification representing diffuse calcification and low attenuation area containing fibrous tissue with possible contrast enhancement as observed in both cases. The walls of the involved sinuses may undergo remodeling and thickening with erosions.

Differential diagnosis includes fibrous dysplasia, osteoid osteoma, osteoblastoma, cementoblastoma, periapical or focal cemento-osseous dysplasia, chronic osteomyelitis, Garre's osteomyelitis and variants of ossifying fibroma, like psammomatous ossifying fibroma. ${ }^{3-6}$

Histopathologically, OFs are usually well circumscribed and partly encapsulated fibro-osseous lesions consisting of abundant cellular fibrous tissues with scattered trabaculae of lamellar woven bone and cementoid masses with osteoblastic rim, while fibrous dysplasia characteristically involves irregulary shaped and woven bone trabaculae or vascularized fibrous stroma in various quantities; they do not involve the osteoblastic rims. ${ }^{12}$ Psammomatoid ossifying fibromas are notable for the presence of bony spicules and mineralized/calcified psammomatoid material. Osteomas are 
well-differentiated benign tumors that predominantly contain lamellar bone; ivory and mature type may contain fibrous tissue. Osteoblastomas contain ample amount of osteoid produced by overabundant osteoblasts. ${ }^{13}$

\section{TREATMENT AND PROGNOSIS}

Treatment of OF is extensive surgical excision to prevent recurrence as there are cases where they are reported to be aggressive and locally destructive. Lesions of paranasal sinuses are removed endoscopically but in most cases they cannot be resected completely due to being in the vicinity of many vital structures, including the skull base. OFs in these areas are, therefore, known for recurrence leading to repeated surgical excision. A close follow-up is, therefore, essential to detect recurrence early. OFs which are in other areas, like mandible and maxilla, can be excised completely and so are less prone to recur.

\section{CONCLUSION}

The diagnosis of OF consists of a combination of clinical, radiological and histopathological criteria. Surgical excision of tumor is the treatment of choice. Transnasal endoscopic excision of tumor from nose and paranasal sinuses is a good approach to deal with intranasal mass. Endoscopes provide panoramic view and vital structures can be identified easily. Endoscopy ensures maximal removal though many times complete removal may be difficult. Attachment of this tumor to vital structures, like eye and skull base, often limits the surgeon to perform a complete removal. There is always a high probability of revision surgeries and this should be well explained to the patient. Continued follow-up is equally important in these cases.

\section{REFERENCES}

1. Reichart PA, Philipsen HP, Sciubba JJ. The new classification of head and neck tumours (WHO) — any changes? Oral Oncol 2006;42:757-58.

2. Yilmaz Ismail, Bal Nebil, Ozluoglu Levent N. Isolated cementoossifying fibroma of the ethmoid bulla-a case report: ENTEar, Nose and Throat Journal 2006;85(5):322-24.

3. Waldron CA. Fibro-osseous lesions of jaw. J Oral Maxillofac Surg 1993;51:828-35.

4. Su L, Waldron CA. Distinguishing features of focal cementosseous dysplasia and cement-ossifying fibroma: A pathologic spectrum of 316 cases. Oral Med Oral Pathol Oral Radiol Endod 1997;84:301-09.

5. Manganaro AM, Ragno JR Jr, Karlis V. Mixed radiolucent/ radiopaque lesions of the mandible. J Oral Maillofac Surg 1997; 55:1456-59.

6. Bowyer JD, Majid MA, Ah-Fat F, et al. Giant cement-ossifying fibroma of maxilla causing proptosis in a young patient. J Pediatr Ophthalmol Stribismus 2001;38:359-62.

7. Brademen G, Werner JA, et al. Cemento-ossifying fibroma of petromastoid region: Case report and review of literature. J Laryngol Otol 1997;111:152-55.

8. Hamner JE, Scofied HH, Cornyn J. Benign fibro-osseous jaw lesions of periodontal membrane origin. An analysis of 249 cases. Cancer 1968;26:861-78.

9. Pindborg JJ, Kramer IRH. Histological typing of odontogenic tumors, jaw cysts and allied lesions. In: International histological classification of tumors. Geneva: WHO 1971:31-34.

10. Kramer IRH, Pindborg JJ, Shear M. Neoplasm and other lesions related to bone. Histologic typing of odontogenic tumors, World Health Organization. Berlin, Springer-Verlag 1992:28-31.

11. Krausen AS, Gulmen S, Zografakis G. Cementomas. Aggresive cement-ossifying fibroma of the ethmoid region. Arch Otolaryngol 1977;103:371-73.

12. Wenig BM, Vinh TN, Smirniotopoulaos JG, et al. Aggressive psammomatoid ossifying fibromas of the sinonasal region: A clinicopathologic study of a distinct group of fibro-osseus lesions. Cancer 1995;76:1155-65.

13. Mohammadi-Araghi H, Haery C. Fibro-osseous lesions of craniofacial bones. The role of imaging. Radiol Clin North Am 1993;31:121-34. 